\title{
Desarrollo del entorno personal de aprendizaje para tutoría e investigación en niveles educativos superiores
}

\section{Staff Development learning environment for tutoring and research in higher education levels}

\author{
John Castevich ${ }^{1}$, Alma Dzib Goodin², Dolores Luna Hogan ${ }^{3}$, Linda Sanders ${ }^{4}$, Kathleen \\ Slovec $^{5}$, Daniel Yelizarov ${ }^{6}$ \\ ${ }^{1}$ Learning y Neuro-Development Research Center, United States (jcastevich@learning.nd.com) \\ ${ }^{2}$ Learning y Neuro-Development Research Center, United States (alma@almadzib.com) \\ ${ }^{3}$ Learning y Neuro-Development Research Center, United States (dlhogan@learning.nd.com) \\ ${ }^{4}$ Learning y Neuro-Development Research Center, United States (LindaSanders@learning.nd.com) \\ ${ }^{5}$ Learning y Neuro-Development Research Center, United States (kslovec@learning.nd.com) \\ ${ }^{6}$ Learning y Neuro-Development Research Center, United States (dyelizarov@ @learning.nd.com)
}

Recibido el 27 de marzo de 2015; revisado el 5 de mayo de 2015; aceptado el 5 de junio de 2015; publicado el 6 de junio de 2015

\section{RESUMEN:}

El concepto de entorno personal se refiere a los sistemas que facilitan a los aprendices a tomar control de manera autónoma de sus aprendizajes. Esto incluye apoyos para determinar sus propias metas, comprensión de contenidos y de los procesos implicados en el proceso de aprendizaje. Usualmente el mayor impulso se ofrece en torno a las nuevas tecnologías, ya que se supone que el alumno es capaz de interactuar de manera efectiva con los lenguajes y entornos tecnológicos, pues más jóvenes las generaciones, más conocimiento tienen del uso y manejo de la información en medios digitales.

Partir de este supuesto, y suponer que el alumno es capaz por sí mismo de encontrar su propio camino, lleva a mucha frustración, especialmente cuando se considera como factor clave las diferencias culturales.

El presente trabajo busca reconocer que existen diferencias culturales en el manejo de herramientas que se consideran usuales como las redes sociales para desarrollar propuestas académicas que permitan apoyar el aprendizaje de los estudiantes y se encuentra que existen factores que pueden potencialmente beneficiar o entorpecer el desarrollo académico de los estudiantes si estas no son consideradas en los programas educativos.

Reconocer dichas diferencias es clave para trabajar en programas de apoyo a los estudiantes, pues una vez que éstos reconocen sus potencialidades y son capaces de generar las estrategias adecuadas a los requerimientos de las nuevas tecnologías, serán más eficientes en su aprendizaje.

PALABRAS CLAVE: ENTORNOS VIRTUALES, EDUCACIÓN SUPERIOR, PLE.
ABSTRACT:
The concept of personal environment refers to systems that facilitate learners to take control of their learning autonomously. This includes support for determining their own goals, understanding of content and processes involved in the learning process. Usually the biggest boost comes in around new technologies, since it is assumed that the student is able to interact effectively with the languages and technological environments, as younger generations, most knowledgeable use and management of information digital media. 
From this assumption and assume that the student is able by itself to find its own path, leading to much frustration, especially when considered as a key factor of cultural differences.

This paper seeks to recognize that there are cultural differences in handling tools that are considered usual as social networks to develop proposals enabling academic support and student learning is that there are factors that can potentially benefit or hinder academic development students if these are not considered in educational programs.

Recognizing these differences is key to working in support programs for students, because once they recognized their potential and are able to generate appropriate to the requirements of new technology strategies will be more efficient in their learning.

\section{KEYWORDS: VIRTUAL ENVIRONMENT,} HIGHER EDUCATION, PLE

\section{ESTRUCTURA DEL ARTÍCULO}

La promesa de un mejor aprendizaje centrado en las nuevas tecnologías comenzó a tener forma hace más de una década gracias al rápido desarrollo de plataformas, aplicaciones y la constante búsqueda de insumos pedagógicos. Sin embargo, a pesar del rápido crecimiento de cursos en línea que pronto se convirtieron en conglomerados conocidos como MOOCs, el aparato Educativo a nivel mundial continuó con el mismo patrón: creando currículos que los estudiantes deben intentar, por lo menos descifrar (Adell, y Castañeda, 2013; Baran, 2013; Solimeno, Mebane, Tornal y Francescato, 2007).

Los nuevos cursos en línea, de cualquier índole requerían habilidades para los que los estudiantes no estaban preparados, además las redes sociales y los buscadores de información, comenzaron a crear un problema de atención que se maximiza aún más cuando se tienen diversas tareas que realizar y metas específicas, básicamente sobre estimulando al sistema cognitivo, carente de habilidades metacognitivas, creando un problema para los estudiantes, en tres grandes momentos, con necesidades diferentes: estudiantes que buscan ingresar a las universidades, estudiantes en proceso de formación, y aquellos que se encuentran en proceso de abandonar los estudios e ingresar al mercado laboral (Ackerman, Dachtera, Pipek y Wulf, 2013; Sendag y Odabash, 2009).

Una alternativa de apoyo ante tal situación, en cualquiera de los niveles, es el aprendizaje centrado en el alumno, el cual es un recurso que usualmente se emplea como proceso paralelo a la Educación formal, bajo el nombre de asesor o mentor, que permite al alumno reconocer sus debilidades para hacerlas fortaleza $\mathrm{y}$ reconocer los contenidos académicos en los que es más apto, con el fin de desarrollar estrategias que le permitan resolver aquellos problemas en los temas en lo que cree tener menos control (An, Shin y Lim, 2009; Laurillard, 2009; Mak, Wiliams y Mackness, 2010; Lazakidou, y Retails, 2010; Wang y Eccles, 2013; León Martínez y Tapia Rangel, 2013).

Sin embargo, a pesar de que estas figuras existen en la Educación Formal, existe el problema de que existe mucha demanda para unos cuantos mentores o asesores y estos restan calidad al trabajo, generando que el alumno termine haciendo lo que puede antes sus necesidades académicas.

Con la llegada de la era digital, este proceso logró la ventaja de volverse asincrónico, y a distancia, aunque vino a complejizar el proceso del aprendizaje, ampliando la cantidad de información y los recursos disponibles para estudiantes y maestros capaces de comprender el papel y las bondades de las nuevas herramientas tecnológicas. Muchas veces la gran cantidad de recursos, crea problemas en los estudiantes que saben usarlos de manera elemental (Dorner, 2012; Reig Hernández, 2013).

Bajo este contexto, el desarrollo de plataformas, materiales y aplicaciones ha sido abundante, particularmente en la última década, buscando crear mecanismos de masificación de la Educación, con el fin de lograr el mayor número de estudiantes al menor costo, rompiendo fronteras y haciendo del aprendizaje una oportunidad al alcance de los dedos, se pasó de la Web 1.0 , a la 2.0 y actualmente se vislumbra la llegada de la tercera generación, con una accesibilidad optima de los contenidos multimedia, todo al alcance de cualquier aparato capaz de sincronizar información desde cualquier parte del mundo (Woo y Reeves, 2007).

Sin embargo, a pesar de todos los cambios de los modelos tecnológicos, mientras la escuela tradicional se centra en el currículo, las nuevas plataformas se centraron en las herramientas, y olvidaron, como siempre ha ocurrido, al estudiante. Dando por hecho que en la era digital, todos eran capaces de leer, escribir o usar un ordenador sin problemas, pero la realidad es que la nueva era trajo consigo alumnos perplejos ante la cantidad de información sin herramientas cognitivas para procesarla (Wolff, 2013; et al., 2013; Sun, et al., 2008). Todo basado en el equipo con el que cuentan desde hace muchos años y producto de la evolución (Dzib Goodin, 2013) Con las cuales intentaban crear 
estrategias capaces de dar respuesta ante sus ambientes (Dzib Goodin, 2012).

Bajo este panorama, surge el modelo que a continuación presentamos, creando estrategias no de enseñanza, sino de aprendizaje que el alumno sea capaz de ejecutar ante cualquier ambiente con el fin de beneficiar su desarrollo.

Pregunta de investigación: ¿Son las herramientas tecnológicas suficientes para afectar de manera positiva el proceso de aprendizaje de los estudiantes?, ¿Cuál es el impacto de la cultura en el aprendizaje basado en nuevas tecnologías?

Hipótesis de trabajo: Las nuevas tecnologías no son suficientes para mejorar las estrategias de aprendizaje, pues dependen directamente de las estrategias metacognitivas de los estudiantes.

\section{EL ESTUDIANTE COMO CENTRO DEL APRENDIZAJE}

Empleando investigación en torno al aprendizaje (Kwon, Hong y Laffey, 2013; Järveia y Hadwin, 2013; Kirschner y Van Merriënboen, 2013; JouneauSion y Sanchez, 2012; Kop, 2011; Kramarski y Michalsky, 2010), centrado en el modelo neurocientífico, se comenzó a elaborar un programa cuyo principal interés es el estudiante, ofreciendo como eje de las actividades situaciones cotidianas que permitieran desarrollar habilidades metacognitivas no desarrolladas o no consolidadas en un grupo de estudiantes provenientes de contextos culturales diferentes, bajo un esquema de que no se iba a enseñar nada, que el alumno no necesitara saber y aplicar en su entorno educativo, $o$ profesional inmediato.

El programa se ofertó en los Estados Unidos, donde muchos de los usuarios son extranjeros, con un manejo pobre del idioma inglés, de las matemáticas y con entornos de aprendizaje diversos, sin objetivos claros de aprendizaje, y con fuentes de información pobres.

Con una breve entrevista se descubrió que los estudiantes tenían muchas ideas en contra de sus propias capacidades y veían los estudios como un trámite y no como una meta. Por lo que se comenzó a ampliar las líneas de investigación para dar respuesta a las necesidades de los usuarios.

A diferencia del Coaching, que centra la interacción del alumno con un guía que se convierte en un motivador, el Entorno Personal de Aprendizaje centra el esfuerzo del alumno en sus propias habilidades, fijando sus propios objetivos de aprendizaje, reconociendo en quienes puede apoyarse para crear redes de información, no solo documental sino personal, reflexionando sobre sus propios recursos cognitivos y con ello creando nuevas metas de aprendizaje, todo ello empleando las ventajas de la educación a distancia, con las ventajas de horarios flexibles, trabajos asincrónicos y organización en el uso y manejo del tiempo (Casquero, 2013 Dabbaghm y Kitsantas, 2013).

\section{RECURSOS TECNOLÓGICOS}

La gestión tecnológica de recursos se convierte en un tema complicado cuando se propone un modelo de Aprendizaje en Línea, ante la variedad de plataformas, y la velocidad en que las actualizaciones se desarrollan, requirió de un análisis minucioso definir los recursos a emplear.

El primer gran paso fue determinar que no se emplearían aplicaciones que se relacionan con Enseñanza, esto con el fin de centrar al usuario en un modelo mucho más personal y sin que tuviera que aprender el uso de las aplicaciones, pues ante sus necesidades no se pueden crear nuevas fuentes de ansiedad.

Además, se debía pensar en aplicaciones usadas desde cualquier lugar del mundo, capaz de actualizarse con el desarrollo de los sistemas operativos principales (IOS y Windows), en nube y con sincronía si fuera posible, gratuita, y con acceso ilimitado; frente a muchas críticas, se tomó la decisión de usar Facebook, Twitter y las bondades que las aplicaciones de Google tiene, servicios que los usuarios conocen, de fácil acceso, con información en nube, abiertos, gratuitos y sobre todo, personalizados, sin embargo, en contra de la idea popular, los estudiantes tienen pobre manejo de éstas aplicaciones, por lo que parte del programa se fijó como meta hacer que los estudiantes usaran las aplicaciones para resolver problemas cotidianos, para que poco a poco se convirtieran en parte de su entorno personal.

\section{PRIMER GRAN PROBLEMA DEL MODELO: LAS DIFERENCIAS CULTURALES}

La primera convocatoria atrajo a un grupo de usuarios que sumó a 119 estudiantes claramente diferenciables: Estudiantes afroamericanos e hispanos (79) de escasos recursos con pobres habilidades tecnológicas, estudiantes hindúes y europeos (40) con recursos económicos, pobreza lingüística pero excelente manejo tecnológico, todos con dificultades especificas en la áreas académicas 
pero todos con el mismo enfoque personal: "Dime lo que tengo que hacer y yo lo hago".

Se desarrollaron estrategias centradas en ellos mismos que les permitieran comprender que no pueden depender del tutor, que tienen habilidades y talentos específicos a partir de sus experiencias y apoyos para el desarrollo de habilidades lingüísticas, no como una materia, sino a partir de actividades centradas en lo que ellos son capaces de realizar, empleando aplicaciones al alcance de sus dedos basado en diversos estudios entre los que se encuentran: Balaji (2010); Alterman y Larusson (2013); Blooma et al. (2013); Eitel et al. (2013).

Una de las herramientas de Google que muchos de ellos no conocían es Google Traductor, misma que les permitía traducir palabras, escucharlas y ampliar su vocabulario. Descubrieron que podían escribir y cotejar la estructura gramatical, sin olvidar que la versión final de su escrito dependía de ellos mismos (Haag et al., 2013).

Los invitamos a escribir pequeñas ideas en el grupo de Facebook, se iniciaron pequeñas competencias, y comenzaron a hablar entre ellos, a compartir conocimientos, a ver las tutorías como algo agradable. Se les comenzó a compartir información sobre los temas que a todos les interesa, y eventualmente, nos permitieron hacer investigación con las experiencia de usuarios y tutores (Hsiao et al., 2013).

Una de las primeras actividades era escribir en tres palabras un poema. Una tarea aparentemente simple donde cada estudiante va agregando dificultad, a partir de la interacción del grupo y de un sentido de competencia.

Las herramientas que continuaron la construcción de su entorno fueron Google especialmente la búsqueda de imágenes, Google Scholar y Wikipedia.

Todas las tareas propuestas, son simples, y se deja que los estudiantes mismos traten de encontrar las respuestas, hasta que se comienzan a dar por vencidos, por lo que la única indicación es preguntar cuando crean que no van a lograr la meta propuesta (Gress et al., 2008; Howe, 2013).

Este punto es clave, la velocidad con que ellos van a buscar apoyo es determinante para permitir la creación de su propio entorno, para ello es necesario que confíen en dicho entorno y que sepan que las herramientas o personas están cerca para aprovechar al máximo el tiempo.

Se encuentran diferencias culturales en este sentido, los hispanos y afroamericanos intentan llegar a la solución de los problemas por si mismos, a pesar de reconocer que les lleva mucho tiempo y que no cuentan con las herramientas o habilidades necesarias. A diferencia, los indoeuropeos son capaces de buscar ayuda rápidamente, pero no confían en sus propias habilidades, por lo que el apoyo es más una confirmación de que hacen lo correcto, estos hallazgos fueron similares a los reportados por Iredale (2006) con deficiencias similares a las reportadas por Jared, Adjei-Boateng y Diteeyont (2013).

Las estrategias que se emplean son simples y buscando que no sean vistas como tareas, si no como retos simples que les permitan crear estrategias, rápidas para dar respuesta a problemas concretos y cotidianos.

Con el uso de aplicaciones que ellos conocen, es posible ir avanzando sobre pasos conocidos hasta llegar al auto reconocimiento de las habilidades y la capacidad de preguntar, pedir apoyo, reconocer las cadenas de tareas necesarias para comprender un problema, planear las respuestas y resolver con éxito un problema, habilidad clave en las materias de ciencia y tecnología Dzib Goodin y Yelizarov, 2013; Wroblewska y Szczepaniak, 2012).

Una de las tareas que permiten mejor analizar las estrategias de resolución de problemas es el juego Cut the Rope, se les pide que resuelvan un reto específico y se pide que anoten cuanto tiempo les toma resolverlo y las estrategias empleadas, cuando son capaces de decir: no creo que esto tenga solución, se les enseña a usar el buscador de Google y una vez que observan cómo resolver el reto, se les pide que digan cómo se sienten y que analicen cuanto tiempo perdieron a pesar de no conocer la forma correcta de resolver el problema, cuando tienen muchos recursos a su alcance que les apoyan en su práctica diaria.

Una vez que las herramientas comienzan a tener sentido para ellos, se crean estrategias para reconocer los pasos para la resolución de problemas, que consisten en el reconocimiento del problema, las variables intervinientes, el planteamiento de estrategias para resolverlo, la ejecución y la medición de los resultados. Con todo ello y las estrategias tecnológicas, se logra usuarios absolutamente independientes, con pensamiento flexible y adaptado ante cualquier situación ya sea académica o de orden común.

\section{SEGUNDO PROBLEMA: EL CONOCIMIENTO DE LAS HERRAMIENTAS}

Los usuarios estaban seguros que conocían las bondades de las herramientas, por ejemplo, todo el mundo sabe que Wikipedia no es una buena fuente 
de información, pero cuando les pedimos que definieran un concepto, se dieron cuenta que es una buena fuente, aunque se les enseñó a reconocer aquellas entradas que requieren de información científica.

Pasaron de usar el $20 \%$ del potencial de Facebook y Twitter a usarlo al $100 \%$, aprendieron a guardar en nube sus lecturas, sin necesidad de perder tiempo en la búsqueda de información, ya que Flipboard es una herramienta gratuita con interacción de muchas fuentes de información.

Descubrieron que YouTube sirve para mucho más que encontrar video para reír, hay lecciones completas, conferencias, resolución a problemas académicos. También reconocieron que en contra de la idea popular, no todas las conferencias de TED son relevantes y diseñaron estrategias personales para criticar la información.

Google Scholar es el mejor buscador para encontrar referencias de todo nivel y solo deben fijar el tipo de información que requieren, aunque en primer lugar reconocieron el tipo de información que necesitan.

\section{CONCLUSIONES}

Las herramientas tecnológicas deben ser vista como apoyos para el aprendizaje, pero nunca podrán sustituir la capacidad de resolución de problemas que se enfrentan en el ambiente cotidiano, natural ya sea académico o profesional. Bajo la idea de que los ordenadores procesan basura si la pregunta no es la correcta, pues dependen de los procesos cognitivos humanos para integrar la información y responder, el modelo de tutoría que busca crear el ambiente personal de aprendizaje, no puede centrarse sólo en las innumerables aplicaciones que se desarrollan todos los días, olvidando las capacidades humanas, sino poner al servicio de la resolución de problemas planteados por los humanos para el avance en todas las áreas de la vida cotidiana (Dzib Goodin y Luna Hogan, 2013; Dzib Goodin, 2013; Ray, 2013; Schoor y Bannert, 2011; Noroozi et al., 2011; Puustinen et al., 2009; Puustinen y Rouet, 2009).

Al mismo tiempo, cuando el alumno no conoce o no confía en sus capacidades, le es muy difícil crear patrones de resolución de problemas viables, porque no sabe que su principal problema es conocer sus propias capacidades (Palacheewa, Suwannathachote y Nilsook, 2012; Pikela y Poikela, 2012; Ng, 2012; Peña, 2013; Olcott , 2013).

Mientras que la Educación Formal se basa en habilidades específicas, este modelo se fundamenta en la capacidad reflexiva para reconocer las respuestas que mejor se adaptan a la resolución de un problema y que en ocasiones deberán variarse, dependiendo del tipo de información que se tenga a la mano (Paechter, Maier y Machter, 2010; Özdemir, 2010; Palm et al., 2009).

Bajo el formato tradicional de los entornos de aprendizaje, toda la información está centrada en las nuevas tecnologías, sin embargo si éstas no se saben usar, o no se entiende cuando se pueden emplear, la información no es relevante.

\section{REFERENCIAS}

Ackerman, MS., Dachtera, J., Pipek, V., y Wulf, V. (2013). Sharing knowledge and expertise: The CSCW view of knowledge management. Computer Supported Cooperative Work, 22, 531-573.

Adell, J., y Castañeda, L. (2013). El ecosistema pedagógico de los PLEs. Pp. 29-52. En: L. Castañeda, y J. Adell (2013) Entornos Personales de Aprendizaje: claves para el ecosistema educativo en red. Alcoy: Marfil

Alterman, R., y Larusson, J. A. (2013). Participation and common knowledge in a case study of student blogging. International Journal of Computer-Supported Collaborative Learning, 8(2), 149-187.

An, H., Shin, S., y Lim, K. (2009). The effects of different instructor facilitation approaches on students' interactions during asynchronous online discussions. Computers y Education, 53(3), 749-760.

Balaji, M. S. (2010). Student interactions in online discussion fórum: Emprirical research from "Media Richness Theory" perspective. Journal of Interactive Online Learning, 9(1), 1-22.

Baran, E. (2013). Connect, participate and learn: Transforming pedagogies in Higher Education. Bulletin of the IEEE Technical Committe on Learning Technology, 15 (1), 912.

Blooma, M. J., Kurian, J. C., Kuan Chua, A. Y., Lian Goh, D. L., y Huong Lien, N. (2013). Social question answering: Analysing knowledge, cognitive processes and social dimension of micro-collaborations. Computers $y$ Education, 69, 109-120.

Casquero, O. (2013). PLE: una perspectiva tecnológica. Pp. 7184. En L. Castañeda, y J. Adell (2013), Entornos Personales de Aprendizaje: claves para el ecosistema educativo en red. Alcoy: Marfil.

Dabbaghm N., y Kitsantas, A. (2013). The role of social media in self-regulated learning. International Journal of Web Based Communities, 9(2), 256-273.

Dorner, H. (2012). Effects of online mentoring in computersupported collaborative learning environments: mentor presence and cognitive engagement. American Journal of Distance Education, 26(3), 157-171.

Dzib Goodin, A., y Yelizarov, D. (2013). Abstract thought: Making simple the complexity. Recuperado de http://learningandneurodevelopmentresearch.blogspot.co m/2013/09/abstract-thought-making-simple.html

Dzib Goodin, A., y Luna Hogan, D. (2013). Globalization and Internationalization: Current future of Higher Education? Recuperado de http://learningandneurodevelopmentresearch.blogspot.co $\mathrm{m} / 2013 / 08 /$ globalization-and-internationalization.html 
Dzib Goodin, A. (2013). La arquitectura cerebral como responsable del desarrollo del proceso de aprendizaje. Revista Mexicana de Neurociencia, 14(2), 81-85.

Dzib Goodin, A. (2013). La evolución del aprendizaje: Más allá de las redes neuronales. Revista Chilena de Neuropsicología, 8(1), 20-25.

Dzib Goodin, A. (2012). General or specific skills: The challenge of Higher Education. The Evolllution. Recuperado de http://www.evolllution.com/program_planning/generalor-specific-skills-the-challenge-of-higher-education/

Eitel, A., Scheiter, L., Schüler, A., NystrOm, M. y Holmqvist, K. (2013). How picture facilitates the process of learning from text: Evidence for scaffolding. Learning and Instruction, 28, 48-63.

Goodyear, P., y Carvalho, L. (2013). The analysis of complex learning environments. En H., Beetham y R., Sharpe (Ed), Rethinking Pedagogy for a Digital Age. New York City: Routledge. Pp. 49-63.

Gress, CLZ. Fior, M., Hadwin, AF., y Winne, PH (2008). Measurement and assessment in computer -supported collaborative learning. Computers in Human Behavior, 26(5), 806-814.

Haag, N., Heppt, B., Stanat, P., Kuhl, P., y Pant, HA. (2013). Second language learners' performance in mathematics: Disentangling the effects of academic language features. Learning and Instruction, 28, 24-34.

Hsiao, YP., Brouns, F., Kester, L., y Sloep, P. (2013). Cognitive load and knowledge sharing in learning networks. Interactive Learning Environments, 21(1), 89-100.

Howe, C. (2013). Scaffolding in context: Peer interaction and abstract learning. Learning, Culture and Social Interaction, 2(1), 3-10.

Iredale, A. (2006). Successful learning or falling promise? A situated evaluation of virtual learning environments. (Pp. 1-10). En D. Whitelock, y S. Wheeler. (2006) Association for Learning Technology. 13th International Conference. Heriot-Watt University, Edinburgh, Scotland, UK.

Jared, K., Adjei-Boateng, E., y Diteeyont, W. (2013). Facilitating active social presence and meaninful interactions in online learning. Education and Information Technologies, 18(4), 597-607.

Järveia, S., y Hadwin, A. (2013). New frontiers: regulating learning in CSCL. Educational Psychologist, 48(1), 2539.

Jouneau-Sion, C., y Sanchez, E. (2012). Preparing schools to accomodate the challenge of web 2.0 technologies. Education and Information Technologies. 18 (2), 265270.

Kirschner, PA., y Van Merriënboen, JJG. (2013). Do learners really know best? Urban Legends in Education. 48 (3), 169-183.

Kop, R. (2011). The challenges to connectivist learning on open online networks: learning experiences during massive open online course. International Review of Research in Open and Distance Learning. 13 (3), 20-38.

Kramarski, B., y Michalsky, T. (2010). Preparing preservice teachers for self-regulated learning in the context of technological pedagogical content knowledge. Learning and Instruction. 20 (5), 434-447.

Kwon, K., Hong, RY., y Laffey, JM. (2013). The educational impact of metacognitive group coordination in computer-supported collaborative learning. Computers in Human Behavior. 29 (4), 1271-1281.
Laurillard, D. (2009). The pedagogical challenges to collaborative technologies. Computer-Supported Collaborative Learning, 4, 5-20.

Lazakidou, G., y Retails, S. (2010). Using computer supported collaborative learning strategies for helping students acquire self-regulated problema-solving skills in mathematics. Computers y Education, 54(1), 3-13.

León Martínez, J. y Tapia Rangel, E. (2013) Educación con TIC para la sociedad del conocimiento. Revista Digital Universitaria, 14(1), 1-12.

Mak, S., Wiliams, R. y Mackness, J. (2010). Blogs and fórums as communication and learning tolos in a MOOC. Pp. 275-285. En Proceedings of the 7th International Conference on Networked Learning 2010. Lancaster: University of Lancaster.

Ng. W. (2012). Can we teach digital natives digital literacy? Computers y Education, 59, 1065-1078.

Noroozi, O., Biemans, HJA., Busstra, MC., Mulder, M. y Chizari, M. (2011). Differences in learning processes between sucessful and less sucessful students in computer-supported collaborative learning in the field of human nutrition and health. Computer in Human Behavior, 27, 309-318.

Olcott Jr., D. (2013). Nuevas líneas de aprendizaje: potenciar el uso de recursos educativos abiertos para reforzar la educación no formal. Revista de Universidad del Conocimiento, 10 (1), 151-169.

Özdemir, S. (2010). 'To err is human, but to persist is diabolical':Loss of organizational memory and elearning projects. Computers y Education, 55, 101-108.

Paechter, M., Maier, B., y Machter, D. (2010). Students' expectations of, and experiences in e-learning: Their relation to learning achievements and course satisfaction. Computer y Education, 54(1), 222-229.

Palacheewa, P., Suwannathachote, P., y Nilsook, P. (2012). Critical issues to be concern in selecting tools for teaching high school computer Project lesson using computer-supported collaborative learning. Creative Education, 3, 11-14.

Palm, M. Sire, S., Bogdanov, E., Gillet, D., y Wild, F. (2009). Mapping web personal learning environments. En 2nd Workshop on Mash-Up Personal Learning Environments. Nice, France.

Peña, I. (2013). El PLE de investigación-docencia: el aprendizaje como enseñanza. Pp.93-110. En L. Castañeda, y J. Adell (2013), Entornos Personales de Aprendizaje: claves para el ecosistema educativo en red. Alcoy: Marfil

Pikela, E., y Poikela, S. (2012). Competence and ProblemBased and Future. The Finnish Society for ProblemBased Learning, ProBell, Robaniemi University of Applied Science, University of Lapland. Finland.

Puustinen, M., y Rouet, JF. (2009). Learning with new technologies: Help seeking and information searching revisited. Computers y Education, 53(4), 1014-1019.

Puustinen, M., Volckaert-Legrier, O., Coquin, D. y Bernicot, J. (2009). An analysis of students' spontaneous computermediated help seeking: A step toward the design of ecologically valid supporting tools. Computers $y$ Education, 53(4), 1040-1047.

Ray, B. (2013). More than just remixing: Uptake and new media composition. Computers and Composition, 30(3), 183196.

Reig Hernández, D. (2013). Describiendo al hiperindividuo, el nuevo individuo conectado. Pp. 21-90. En D. Reig, y L. F. Vílchez (2013), Los jóvenes en la era de la 
hiperconectividad: tendencias, claves y miradas. Madrid: Fundación Telefónica- Fundación Encuentro.

Schoor, C., y Bannert, M. (2011). Motivation in a computersupported collaborative learning scenario and its impacto $\mathrm{n}$ learning activities and knowledge acquisition. Learning and Instruction, 21(4), 560-573.

Sendag, S., y Odabash, H. F. (2009). Effects on an online problema based learning course on content knowledge acquisition and critical thinking skills. Computers $y$ Education, 53(1), 132-141.

Solimeno, A. Mebane, M. E., Tornal, M., y Francescato, D. (2007). The influence of students and teachers characteristics on the efficacy of face to face and computer supported collaborative learning. Computer $y$ Education, 51(1), 109-128.

Sun, P. C., Tsai, R. J., Finger, G., Chen, Y., y Teh, D. (2008). What drives a successful e-learning? An empirical investigation of the critircal factors influencing learner satisfaction. Computers y Education, 50(4), 1183-1202.

Voogt, J. Erstad, O., Dede, C., y Mishra, P. (2013). Challenges to learning and Schooling un the digital networked world of the 21st century. Journal of Computer Assited Learning, 29(5), 403-413.

Wang, M. T., y Eccles, J. S. (2013). School context, achievement motivation, and academic engagement: A longitudinal study of school engagemente using a multidimensional perspective. Learning and Instruction, $28,12-23$.

Wolff, W. (2013) Interactivity and the invisible: What counts as writing in the age of the web 2.0. Computers and Composition, 30(3), 211-225.

Woo, Y. y Reeves, T. C. (2007). Meaningful interaction in web -based learning: A social constructivist interpretation. The Internet and Higher Education, 10(1), 15-25.

Wroblewska, I., y Szczepaniak, J. (2012). Problem solving and learning mathematics. (Pp. 90-96). En E. Pikela y S. Poikela. Competence and Problem-Based and Future. The Finnish Society for Problem-Based Learning, ProBell, Robaniemi University of Applied Science. Finland: University of Lapland. 